\title{
Biodiversidad marina en Magallanes: equinodermos del Pabellón de Colecciones Edmundo Pisano V.
}

\author{
Marine biodiversity in Magallanes: echinoderms \\ from the Edmundo Pisano V. Collection
}

Erika Mutschke ${ }^{1}$, Carlos Ríos ${ }^{1}$, Cristian Aldea $^{2}$, Américo Montiel $^{1} \&$ Fernanda Silva $^{1}$

\section{Resumen}

Echinodermata es uno de los taxa más conspicuos en las comunidades marinas del cono austral de Sudamérica y Antártica. Numerosas expediciones extensivas e intensivas en la región han permitido la recolección de un número importante de ejemplares de este taxa, los cuáles están depositados en el Pabellón de Colecciones Biológicas Prof. Edmundo Pisano V. del Instituto de la Patagonia, Universidad de Magallanes (PCEP). En este trabajo se proporciona el primer reporte detallado de las especies, familias y órdenes de Echinodermata incluidos hasta la fecha en el PCEP. La lista catalogada de los ejemplares presentes incluye a 8.589 individuos pertenecientes a las cinco clases existentes de Echinodermata (Crinoidea, Asteroidea, Ophiuroidea, Echinoidea y Holothuroidea). En términos de familia por clase, Asteroidea es la mejor representada con 24 familias. En función del número de especies por familia, Ophiuridae, Asteriidae, y Odontasteridae son las familias mejor representadas en el PCEP. La colección incluye un total de 768 registros de ingreso. Esto incluye a 67 especies de equinodermos propios de Magallanes $(56 \%$ del total de especies reportadas para la región) y 56 especies antárticas (7,3\% del total reportado para esta área). Sólo se ha registrado una especie endémica para Magallanes, el asteroideo Myxoderma qawashqari (Moyano \& Larraín Prat, 1976). Se sugiere la necesidad de continuar con el registro, validación taxonómica y determinación de la riqueza específica de los equinodermos en particular $y$ del bentos en general, presentes en los fiordos y canales australes, considerando la importancia ecológica y biogeográfica de la región. La importancia de mantener y conservar Salas de Colecciones o de Referencias es fundamental dado al papel que juegan en el seguimiento de patrones de impacto externo y de los cambios ambientales sobre la biodiversidad.

\section{Palabras Clave:}

Echinodermata, Asteroidea, Echinoidea, Crinoidea, Holothuroidea, Ophiuroidea, subantártica, estrecho de Magallanes

\section{Abstract}

The Echinodermata (Echinoderms) is one of the most conspicuous phylum in the marine communities around the southern cone of South America and Antarctica. Many extensive and intensive expeditions in the area have collected an important number of specimens within this phylum. These have been deposited and stored in the Professor Edmundo Pisano V. Biological Collection Room at Instituto de la Patagonia, Universidad de Magallanes (PCEP). This paper presents the first detailed report of the species, families and orders within Echinodermata deposited up until March 2016 in the PCEP. The catalogue of specimens includes 8,589 individuals belonging to the five Echinodermata classes (Crinoidea, Asteroidea, Ophiuroidea, Echinoidea and Holothuroidea). In terms of family per class, Asteroidea is the best represented with 24 families. As a function of the number of

\footnotetext{
1 Laboratorio de Hidrobiología, Instituto de la Patagonia, Universidad de Magallanes. $\longrightarrow$ erika.mutschke@umag.cl

2 Facultad de Ciencias, Universidad de Magallanes
} 
number of species per family, Ophiuridae, Asteriidae, and Odontasteridae are the best represented families in the PCEP. The collection is based on 768 admittance records. This includes 67 Echinoderm species from Magallanes $(56 \%$ of the total number of species reported for the area) and 56 Antarctic species (7.3\% of the total reported for this area). Only one species endemic to Magallanes, the Asteroidea Myxoderma qawashqari (Moyano \& Larraín Prat, 1976) has been recorded.

The need to continue the recording, taxonomic validation and assessment of the specific richness of Echinodermata in particular, and the benthos present in the austral fjords and channels in general is recommended, given the ecological and biogeographical importance of the region. The importance of keeping and conserving collection or reference collection rooms is underlined given their role in the monitoring of the impact of external pressures and environmental change on the biodiversity of the region.

\section{Key Words:}

Echinodermata, Asteroidea, Echinoidea, Crinoidea, Holothuroidea, Ophiuroidea, Subantarctic, Straits of Magellan.

\section{INTRODUCCIÓN}

Echinodermata es uno de los taxa más conspicuos en las comunidades marinas del cono austral de Sudamérica (Mutschke, 2006) y Antártica (Jossart et al. 2015). Su presencia ha sido registrada en ambientes sublitorales someros y profundos del sistema de fiordos y canales australes, incluyendo el estrecho de Magallanes (Mutschke \& Ríos, 2006), ambientes intermareales de bloques y canto (Ríos et al. 2005) y en sustratos biogénicos (Ríos et al. 2007). La representatividad del Phylum está dada por todos los órdenes reconocidos hasta la fecha (WoRMS, 2016).

Varios estudios extensivos (CIMAR-Fiordos) e intensivos (e.g. R/V Victor Hensen, 1994) han permitido la recolección de equinodermos en prácticamente toda la región austral comprendida entre los $41^{\circ} \mathrm{S}-56^{\circ} \mathrm{S}$, además de sectores incluidos en el Arco de Scotia, Península Antártica y Mar de Weddell.

Con el material recolectado se ha ido construyendo una colección de referencias instalada en el "Pabellón de Colecciones Biológicas Prof. Edmundo Pisano V." (PCEP) del Instituto de la Patagonia, Universidad de Magallanes (www. coleccionesbiologicasidep.cl).

En este trabajo, proporcionamos un detallado reporte de las especies, familias y órdenes de Echinodermata incluidos hasta la fecha en el Pabellón de Colecciones recolectados en la Región biogeográfica de Magallanes (sensu Longhurst, 1998) y en zonas subantárticas y Antárticas. La información que se entrega es fundamental para desarrollar estudios sobre biodiversidad y biogeografía aún pendientes para la zona austral de Sudamérica y además destaca el valor intrínseco de las salas de colecciones biológicas.

\section{MATERIALES Y MÉTODOS}

El inventario detallado sobre los equinodermos se basa en especímenes recolectados tanto en las zonas intermareales como del sublitoral de fondos duros y blandos, típicos de la región Magallánica de zonas someras y profundas. También se incluye información sobre la fauna de equinodermos recolectada en el Arco de Scotia y Mar de Weddell, Antártica.

En la mayoría de los casos, los especímenes intermareales se recolectaron por medios manuales. Los de aguas someras se obtuvieron por diversas técnicas de buceo, mientras que las profundas en su mayoría por medio de una rastra Agassiz (AGT) modificada o bien con dragas Smith -McIntyre .

El material proviene de las Campañas Victor Hensen, 1994 (Arntz \& Gorny, 1996); Campo de Hielo Sur, 1995 (CONA, 1996); Crucero CIMAR 2 Fiordos, 1996 (CONA, 1997); ANT XIII/5, 1996 (Fahrbach \& Gerdes, 1997); Crucero CIMAR 3 Fiordos, 1997 (CONA, 1998); ANT XV/3, 1998 (Arntz \& Gutt, 1999), Crucero CIMAR 7 Fiordos, 2001 (CONA, 2002); ANT XIX/5, 2002 (Arntz \& Brey, 2003); Crucero CIMAR 8 Fiordos, 2002 (CONA, 2003); Crucero CIMAR 15 Fiordos, 2009 (2010); Crucero CIMAR 16 Fiordos, 2010 (CONA, 2011); Crucero CIMAR 20 Fiordos, 2014 (CONA, 2015).

Los organismos recolectados en campañas anteriores al año 2000 fueron fijado en formalina al $4 \%$ neutralizado con sal de bórax y luego se 


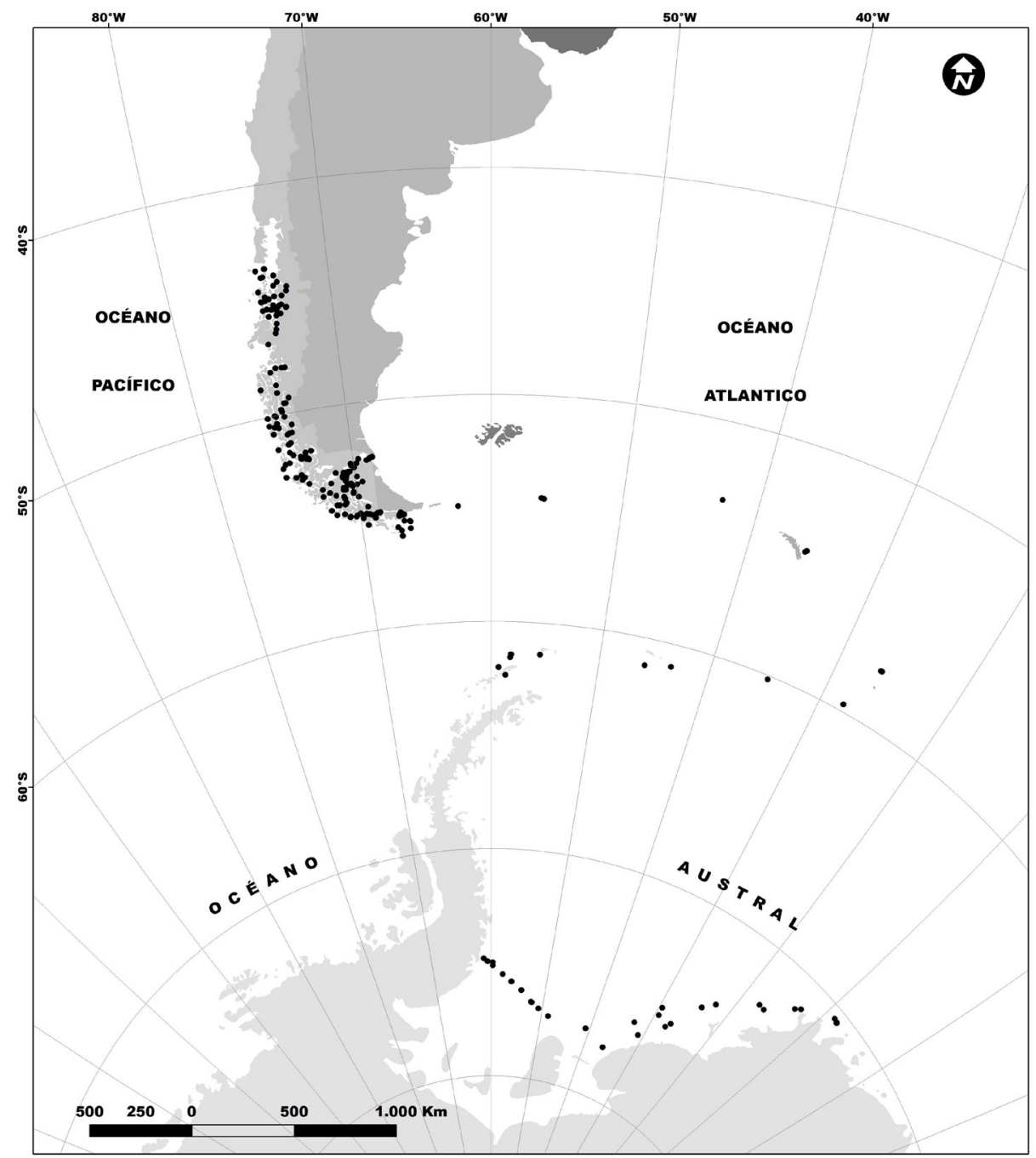

Fig. 1. Lugares de recolección de los ejemplares de equinodermos depositados en Pabellón de Colecciones Biológicas Prof. Edmundo Pisano V. del Instituto de la Patagonia, Universidad de Magallanes (Región de Magallanes y Antártica Chilena).

traspasados a alcohol de $75^{\circ}$. Posterior al año 2000, fueron mantenidos siempre en alcohol.

Todo el material ha sido identificado al nivel taxonómico más bajo posible, utilizando los trabajos de Clark \& Downey (1992), Tommasi (1999), Hendler et al. (1995), Pawson et al. (2010), Bernasconi (1966, 1967, 1968 y 1970), Mutschke \& Mah (2009). Parte de los especímenes han sido revisados por especialistas en algunos grupos.

\section{RESULTADOS}

La distribución de los sitios de muestreo en donde se han recolectado los equinodermos depositados en la el PCEP, incluye, en términos generales, desde sectores del Pacífico Sur Oriental de la Región Biogeográfica Magallánica, parte del Arco de Scotia y Mar de Weddell (Fig. 1). La mayoría de los lugares de muestreo se localizan entre los $41^{\circ} \mathrm{S}$ $-56^{\circ} \mathrm{S}$ (Sur del Golfo de Penas hasta el archipiélago del Cabo de Hornos) dentro del cono sur de América del Sur.

Las especies de Echinodermata incluidas en la colección se obtuvieron a partir de muestras recolectadas en diferentes tipos de sustratos, incluyendo macroalgas (e.g. Macrocystis pyrifera), 


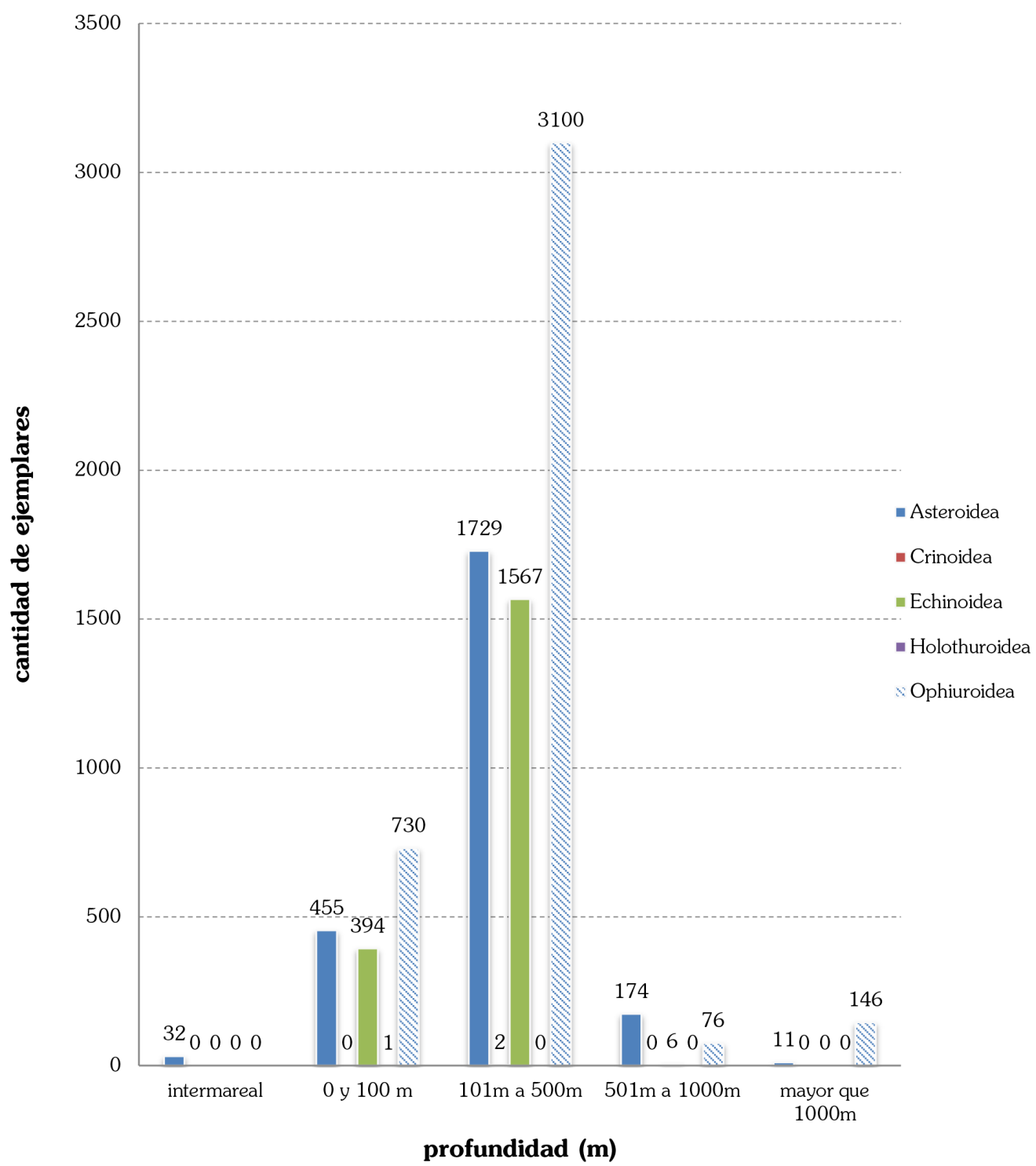

Fig. 2. Número de ejemplares depositados en Pabellón de Colecciones Biológicas Prof. Edmundo Pisano V., separados por profundidad y taxón.

fondos blandos (limos y arcillas frente a glaciares) o duros (roca, bloques y cantos). En general, las especies presentan un rango batimétrico amplio, con una representación máxima de especies obtenidas en profundidades que varían entre 101 - $500 \mathrm{~m}$ (Fig. 2).

La lista catalogada de los ejemplares presentes en la el PCEP, incluye 8.589 individuos pertenecientes a las cinco clases existentes de Echinodermata (Crinoidea, Asteroidea, Ophiuroidea, Echinoidea y Holothuroidea).
Hasta la fecha, la colección incluye 768 registros de ingreso, correspondiendo a un total de 114 especies de las cinco clases de equinodermos (Tabla 1).

En términos de familia por clase, Asteroidea es la mejor representada con 24 familias. Le siguen las clases Echinoidea y Ophiuroidea, ambas con 7 familias. Las clases Holothuroidea y Crinoidea no superan las 2 familias. Específicamente Crinoidea está representada solo por 1 especie, recolectada en una estación localizada en el estrecho de Ma- 
Tabla 1. Lista general de especies de Echinodermata y clases correspondientes que se han recolectado en las regiones de Magallanes y Antártica, depositadas en el Pabellón de Colecciones Biológicas Prof. Edmundo Pisano V. del Instituto de la Patagonia, Universidad de Magallanes.

\begin{tabular}{|c|c|c|c|}
\hline Especie & clase & Especie & clase \\
\hline Acodontaster capitatus & AST & Leptychaster flexuosus & AST \\
\hline Acodontaster conspicuus & AST & Leptychaster magnificus & AST \\
\hline Acodontaster elongatus & AST & Leptychaster melchiorensis & AST \\
\hline Acodontaster hodgsoni & AST & Lophaster gaini & $A S T$ \\
\hline Acodontaster sp. & AST & Lophaster stellans & AST \\
\hline Anasterias antarctica & AST & Cheiraster (Luidaster) gerlachei & AST \\
\hline Anasterias sp. & AST & Lysasterias digitata & AST \\
\hline Anteliaster australis & $A S T$ & Lysasterias perrieri & AST \\
\hline Asterina fimbriata & AST & Macroptychaster accrescens & AST \\
\hline Diplodontias singularis & AST & Myxoderma qawashqari & $A S T$ \\
\hline Bathybiaster loripes & $A S T$ & Notasterias armata & AST \\
\hline Calyptraster tenuissimus & AST & Notioceramus anomalus & AST \\
\hline Ceramaster grenadensis patagonicus & AST & Odontaster meridionalis & AST \\
\hline Cheiraster (Luidiaster) planeta & AST & Odontaster penicillatus & AST \\
\hline Cheiraster (Luidiaster) antarcticus & AST & Odontaster $s p$ & AST \\
\hline Chitonaster johannae & AST & Paralophaster antarticus & AST \\
\hline Cosmasterias lurida & $A S T$ & Paralophaster goodfroyi asperatus & AST \\
\hline Ctenodiscus procurator & AST & Patiriella fimbriata & AST \\
\hline Cuenotaster involutus & AST & Peribolaster folliculatus & AST \\
\hline Cycethra verrucosa & $A S T$ & Perkanaster aurorae & AST \\
\hline Cycethra verrucosa mawsoni & $A S T$ & Perkanaster sladeni sladeni & AST \\
\hline Diplasterias brucei & AST & Perknaster falklandica & $A S T$ \\
\hline Diplopteraster clarki & AST & Poraniopsis echinaster & $A S T$ \\
\hline Ganeria falklandica & $A S T$ & Poraniopsis mira & AST \\
\hline Ganeria hahni & AST & Pseudarchaster discus & AST \\
\hline Porania antarctica & AST & Psilaster charcoti & AST \\
\hline Henricia obesa & AST & Pteraster affinis & AST \\
\hline Henricia parva & $A S T$ & Pteraster stellifer & AST \\
\hline Henricia smilax & AST & Echinaster hirsuta & AST \\
\hline Henricia sp. & AST & Solaster regularis & AST \\
\hline Henricia studeri & AST & Solaster regularis subarcuatus & AST \\
\hline
\end{tabular}


Continuación Tabla 1

\begin{tabular}{|c|c|c|c|}
\hline Especie & clase & Especie & clase \\
\hline Hippasteria phrygiana & AST & Stichaster striatus & $A S T$ \\
\hline Hippasteria sp. & AST & Vemaster sudatlanticus & $A S T$ \\
\hline Hymenaster $s p 1$ & AST & Promachocrinus sp & CRI \\
\hline Kampylaster incurvatus & AST & Abatus sp. & ECHI \\
\hline Labidiaster annulatus & AST & Arbacia dufresnei & ECHI \\
\hline Labidiaster radiosus & AST & Austrocidaris canaliculata & $E C H I$ \\
\hline Brisaster moseleyi & ECHI & Ophiactis asperula & $\mathrm{OPHI}$ \\
\hline Loxechinus albus & ECHI & Ophiactis sp. & $\mathrm{OPHI}$ \\
\hline Pseudechinus magellanicus & ECHI & Ophiocten amitinum & $\mathrm{OPHI}$ \\
\hline Tripylaster philippii & ECHI & Ophiomitrella falklandica & $\mathrm{OPHI}$ \\
\hline Athyonidium chilensis & $H O L$ & Ophiomitrella ingrata & $\mathrm{OPHI}$ \\
\hline Chiridota pisanii & $\mathrm{HOL}$ & Ophiomyxa vivipara & OPHI \\
\hline Hemioedema spectabilis & $H O L$ & Ophionotus victoriae & $\mathrm{OPHI}$ \\
\hline Pseudocnus dubiosus leoninus & $H O L$ & Ophioperla koehleri & $\mathrm{OPHI}$ \\
\hline Psolus patagonicus & $\mathrm{HOL}$ & Ophiophragmus sp. & OPHI \\
\hline Amphioplus peregrinator & OPHI & Ophioplocus incipiens & $\mathrm{OPHI}$ \\
\hline Amphiura belgicae & $\mathrm{OPHI}$ & Ophioscolex nutrix & $\mathrm{OPHI}$ \\
\hline Amphiura joubini & $\mathrm{OPHI}$ & Ophiosteira echinulata & OPHI \\
\hline Amphiura proposita & $\mathrm{OPHI}$ & Ophioscolex nutrix & $\mathrm{OPHI}$ \\
\hline Astrotoma agassiziii & $\mathrm{OPHI}$ & Ophiura lymani & $\mathrm{OPHI}$ \\
\hline Gorgonocephalus chilensis & $\mathrm{OPHI}$ & Ophiura (Ophiuroglypha) carinifera & OPHI \\
\hline Homalophiura sp. & $\mathrm{OPHI}$ & Ophiura (Ophiuroglypha) lymani & $\mathrm{OPHI}$ \\
\hline Ophiolimnia antarctica & $\mathrm{OPHI}$ & Ophioplinthus brevirama & $\mathrm{OPHI}$ \\
\hline Ophiacantha sp. cf. pentactis & $\mathrm{OPHI}$ & Ophioplinthus gelida & $\mathrm{OPHI}$ \\
\hline Ophiacantha vivipara & OPHI & Ophioplinthus sp. & $\mathrm{OPHI}$ \\
\hline Ophiacantha rosea & OPHI & & \\
\hline
\end{tabular}

$\mathrm{AST}=$ Asteroidea; $\mathrm{ECHI}=$ Echinodermata $\mathrm{CRI}=$ Crinoidea; $\mathrm{OPHI}=$ Ophiuroidea $; \mathrm{HOL}=$ Holothuroidea

gallanes.

Las familias más numerosas en términos de número de invididuos en los registros del PCEP son Ophiuridae (3.311 ejemplares catalogados), Temnopleuridae (1.373 ejemplares), Ctenodiscidae (817 especímenes). Las familias Schizasteridae y Goniopectinidae están representadas por 458 y 447 ejemplares, respecti- vamente. Las familias Asteriidae, Ophiactidae, Gorgonocephalidae, Poranidae y Astropectinidae comprenden entre 329 - 164 individuos. 16 familias aportan entre 11 y 87 individuos y 14 familias menos de 10 (Fig. 3).

En función del número de especies por familia, Ophiuridae, Asteriidae, y Odontasteridae son las familias mejor representadas en la colec- 


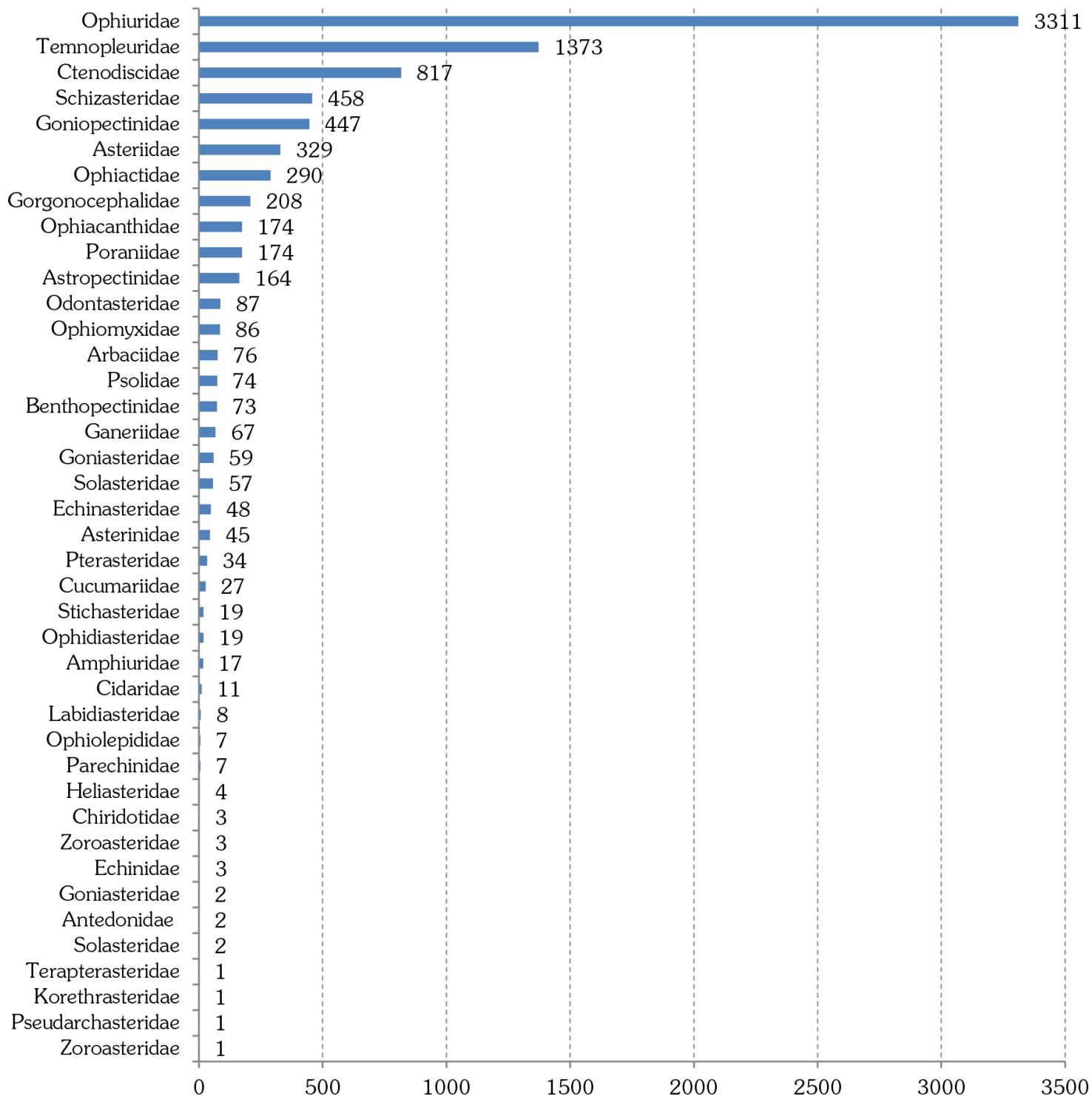

Fig. 3. Número de especímenes existentes en el Pabellón de Colecciones Biológicas Prof. Edmundo Pisano V., asociados a cada familia de equinodermos.

ción con 12, 11 y 10 especies, respectivamente (Fig. 4). Las restantes familias cuentan con una representación específica que varía entre 1 y 8.

La especie más numerosa en los registros es Ophiocten amitinum (20,4\% del material total), Pseudechinus magellanicus (15,6\%), Ctenodiscus procurator $(14,5 \%)$ seguida por Ophiuroglypha lymani (12,7\%), Tripylaster philippii (3,7\%). Las restantes especies registradas representan todos valores menores al $2 \%$.

\section{DISCUSIÓN}

Este trabajo proporciona una visión de conjunto sobre los equinodermos existentes en el PCEP y referidos geográficamente a la región de fiordos y canales australes comprendidos entre el sur de Chiloé y el Cabo de Hornos.

De acuerdo al criterio de clasificación geográfica empleado por Pérez-Rufaza et al. (2013), para la Provincia Magallánica (Puerto 


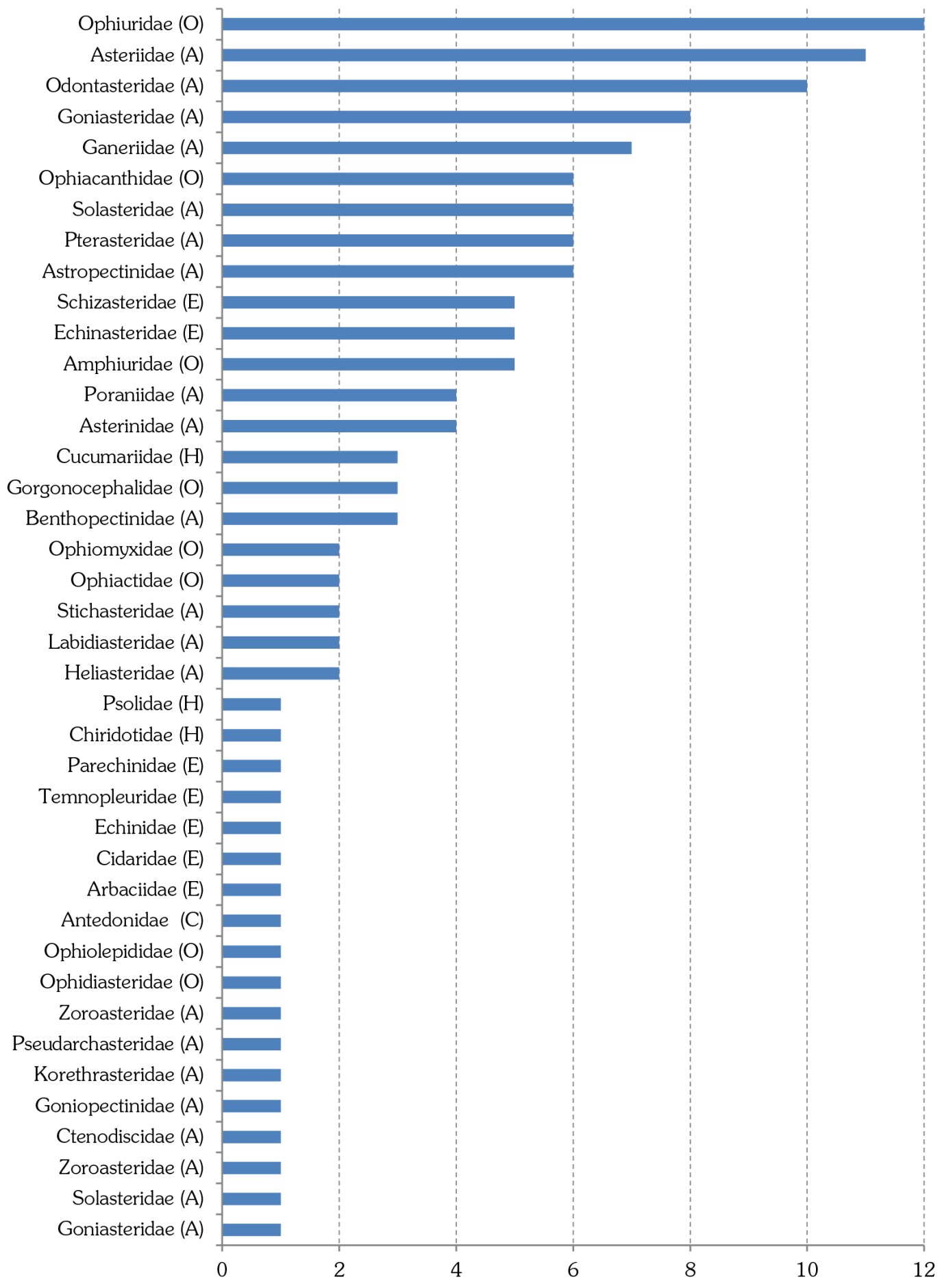

Fig. 4. Número de especies de equinodermos por familia depositados en el Pabellón de Colecciones Biológicas Prof. Edmundo Pisano V. $\mathrm{O}=$ Ophiuroidea; $\mathrm{A}=$ Asteroidea;

$\mathrm{E}=$ Echinoidea; $\mathrm{H}=$ Holothuroidea; $\mathrm{C}=$ Crinoidea. 


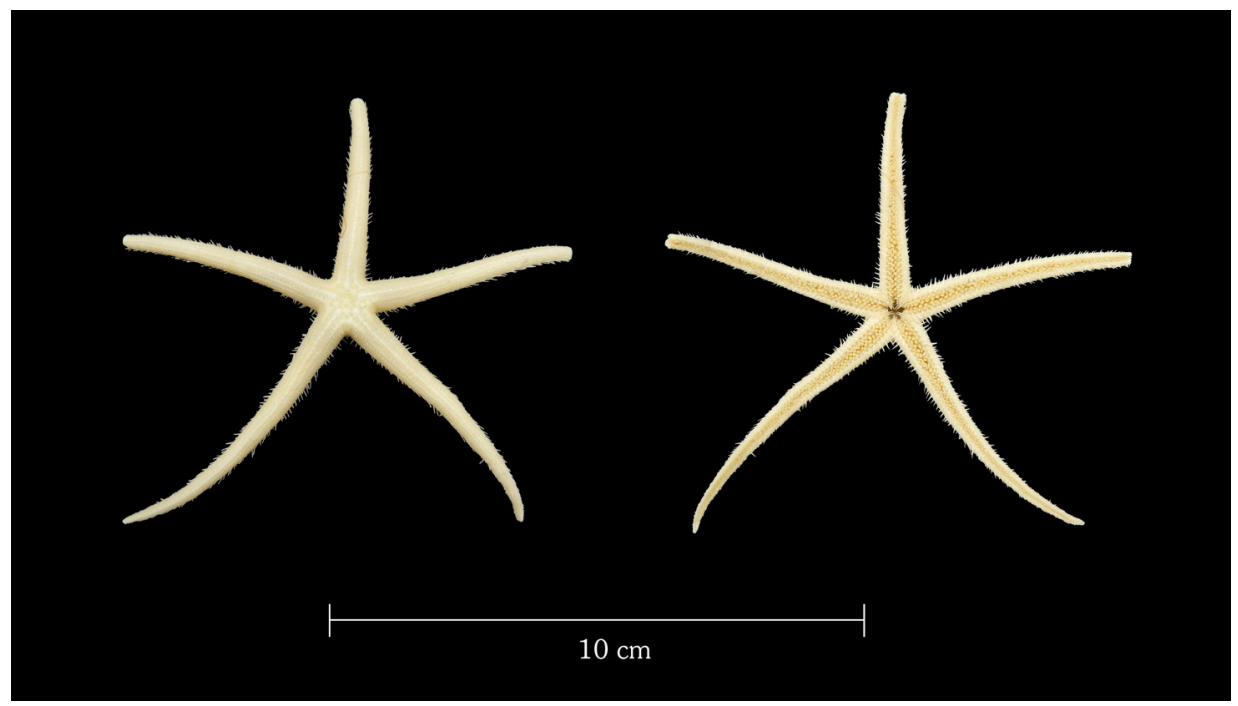

Fig. 5. Myxoderma qawashqari (Moyano \& Larraín Prat, 1976), única especie de asteroideo endémico para Magallanes, depositado en el Pabellón de Colecciones Biológicas Prof. Edmundo Pisano V.

Montt a Cabo de Hornos) se han reportado un total de 119 especies de equinodermos, mientras que para la Provincia Perú-Chilena (Puerto Montt al norte del Perú) se han registrado 44 especies. Brattström \& Johansen (1983) reportan un total de 47 especies de equinodermos presentes en aguas someras de Chile, entre Arica y Cabo de Hornos. Mutschke \& Ríos (2006) reportan sólo para el estrecho de Magallanes un total de 38 especies de equinodermos. De acuerdo al SCAR MarBIN (Griffith et al. 2011), en Antártica se han registrado 728 especies.

En relación con el total de equinodermos indicado por Pérez-Rufaza et al. (2013) solamente para Magallanes (119 especies), el PCEP contiene un $56 \%$ del total registrado para el área (67 especies), mientras que en términos de la representación relacionada con el grupo en Antártica, se han ingresado 53 especies que representan un $7,3 \%$ de las especies validadas para la Antártica (728). Los valores mencionados indican la necesidad de continuar con el registro, validación taxonómica y determinación de la riqueza específica de los equinodermos presentes en los fiordos y canales australes, considerando la importancia ecológica y biogeográfica de la región en términos de riqueza de especie de este grupo tanto en comparación con la Antártica como con zonas al norte de Magallanes.
El endemismo para equinodermos en esta región es notablemente bajo, con sólo una especie exclusiva para Magallanes, el asteroideo Myxoderma qawashqari (Moyano \& Larraín Prat, 1976) (Fig. 5). El registro que se mantiene en el Pabellón de Colecciones proviene del mismo lugar en donde fue capturada originalmente (canal Moraleda).

Finalmente, se debe destacar la importancia de mantener y conservar Salas de Colecciones o de Referencias por su aporte al estudio de la biodiversidad y a los procesos formativos en distintos niveles educacionales, ya sea en escalas regionales como globales (Wilcox \& Murphy, 1985; Balmford et al, 2005; Hannah, 2015; Krishtalka \& Humphrey, 2000; Mikkelsen \& Cracraft, 2001). En este escenario, las colecciones biológicas representan un valioso resguardo de especímenes, incluyendo la mantención de estos en términos de recursos genéticos. La mantención y continuidad de salas de colecciones biológicas, es particularmente relevante en ecosistemas marinos, en donde los efectos de cambios naturales o antrópicamente inducidos, pueden ser ignorados con facilidad. Específicamente, la Región Magallánica, cubre desde los $45^{\circ}$ a los $56^{\circ} \mathrm{S}$ por el océano Pacífico, con una extensión superior a los $84.000 \mathrm{~km}$ de línea costera (Silva \& Palma, 2008). Aquí se genera un complejo sistema de canales, fiordos, 
con fuertes efectos sobre la biodiversidad del área.

\section{AGRADECIMIENTOS}

La correcta aplicación de principios taxonómicos en la identificación de los equinodermos de la zona de fiordos y canales australes ha sido posible sólo gracias al invaluable apoyo de numerosos expertos. Valoramos los extraordinarios aportes del Dr. Igor Smirnov, quien dedicó tiempo a revisar las identificaciones preliminares y a determinar específicamente material de Ophiuroidea. Al Dr. Alberto Larraín por el impulso inicial para que EMO tome el estudio de los equinodermos australes. Agradecemos a la Dra. Cindy Ahearn ( $\dagger$ ) quien, junto al Dr. David Pawson, abrieron las puertas de la colección de asteroideos del Smithsonian National Museum of Natural History, Washington. Al Dr. Alejandro Tablado, quien además de colaborar gentilmente en la identificación de material, facilitó la revisión de las valiosas colecciones de equinodermos de la Dra. Irene Bernasconi $(\dagger)$ del Museo Argentino de Ciencias Naturales "Bernardino Rivadavia", Buenos Aires. A los Dres. Pedro Báez y Sergio Letelier, quienes facilitaron el trabajo en el Museo Nacional de Historia Natural, Santiago de Chile, especialmente en el año 2012. Al Dr. Claude de Broyer, por permitir la revisión de material en Bruselas. Nuestro reconocimiento al Prof. Dr. HC Wolf E. Arntz (AWI), por su gran aporte para el fortalecimiento de los estudios sobre el bentos de la Región Magallánica, normalmente ignorado. Adicionalmente, al Dr. Jim Mc Adam por sus sugerencias al abstract.

\section{LITERATURA CITADA}

Arntz, W. E., \& J. Gutt. (1999). The Expedition ANTARKTIS XV/3 (EASIZ II) of RV "Polarstern" in 1998. Berichte zur Polarforschung, 301, 1-229.

Arntz, W. E., \& M. Gorny. (1996). Cruise report of the Joint Chilean-German-Italian "Victor Hensen" Campaign in 1994. Reports on Polar and Marine Research, 190, 1-113.

Arntz, W. E., \& T. Brey. (2003). Expedition ANTARKTIS XIX/5 (LAMPOS) of RV "Polarstern" in 2002. Reports on Polar and Marine Research, 462, 1-122.
Balmford, A., Bennun, L., Ten Brink, B., Cooper, D., Côté, I. M., Crane, P.,...Gregory, R. D. (2005). The convention on biological diversity's 2010 target. Science, 307.

Bernasconi, I. (1966). Los equinoideos y asteroideos colectados por el buque oceanografico R/V" Vema": frente a las costas Argentinas, Uruguayas y sur de Chile. Revista Museo Argentino Ciencias Nataturales Zoología, 9(7), 147-175.

Bernasconi, E. (1968). Equinodermos de las islas Marion y Príncipe Eduardo con descripción de una nueva especie de Ofiuroideo. Physics, 28, 55-58.

Bernasconi, I. (1967). Algunos Asteroideos abisales del oeste de Chile. Physis, 26, 443-449.

Bernasconi, I. (1970). Equinodermos antárticos. II. Asteroideos. 3. Asteroideos de la extremidad norte de la Península Antártica. Revista del Museo Argentino de Ciencias Naturales, 9(10), 211-281.

Brattström, H., \& Johanssen, A. (1983). Ecological and regional zoogeography of the marine benthic fauna of Chile: Report no. 49 of the Lund University Chile Expedition 1948-49. Sarsia, 68(4), 289-339.

Clark, A. M., \& Downey, M. E. (1992). Starfishes of the Atlantic. Chapman \& Hall.

Comité Oceanográfico Nacional. (1996). Crucero de Investigación Científica Marina a los Fiordos y Canales adyacentes a Campos de Hielo Sur, 1995. Informe de Crucero. Ed. Comité Oceanográfico Nacional, Valparaíso.

Comité Oceanográfico Nacional. (2003). Resultados preliminares Crucero CIMAR 8 Fiordos (2002). Ed. Comité Oceanográfico Nacional, Valparaíso.

Comité Oceanográfico Nacional. (2010). Resultados preliminares Crucero CIMAR 15 Fiordos (2009). Ed. Comité Oceanográfico Nacional, Valparaíso.

Comité Oceanográfico Nacional. (2015). Resultados preliminares Crucero CIMAR 20 Fiordos (2014). Ed. Comité Oceanográfico Nacional, Valparaíso.

Comité Oceanográfico Nacional. (1997). Resultados preliminares Crucero CIMARFiordo 2 (1996). Informe de Crucero. Ed. Comité Oceanográfico Nacional, Valparaíso. 
Comité Oceanográfico Nacional. (1998). Resultados preliminares Crucero CIMARFiordo 3 (1997). Informe de Crucero. Ed. Comité Oceanográfico Nacional, Valparaíso. Comité Oceanográfico Nacional. (2002). Resultados preliminares Crucero CIMARFiordo 7 (2001). Informe de Crucero. Ed. Comité Oceanográfico Nacional, Valparaíso. Comité Oceanográfico Nacional. (2011). Resultados preliminares Crucero CIMAR 16 Fiordos 16 (2010). Ed. Comité Oceanográfico Nacional, Valparaíso.

Farhbach E., \& Gerdes, D. (1997). Die Expedition ANTARKTISXIII/4-5 desForschungsschiffes Polarstern 1996. Reports on Polar and Marine Research, 239, 1-126.

Griffiths, H. J., Danis, B., \& Clarke, A. (2011). Quantifying Antarctic marine biodiversity: The SCAR-MarBIN data portal. Deep Sea Research Part II: Topical Studies in Oceanography, 58(1), 18-29.

Hannah, L. (2015). Climate Change Biology $\left(2^{\circ}\right.$ ed.). United Kingdom: Editorial Elsevier.

Hendler, G., Miller, J. E., Pawson, D. L., \& Kier, P. M. (1995). Sea stars, sea urchins, and allies: echinoderms of Florida and the Caribbean. (No. Sirsi) i9781560984504).

Jossart, Q., Moreau, C., Agüera, A., De Broyer, C., \& Danis, B. (2015). The Register of Antarctic Marine Species (RAMS): a tenyear appraisal. ZooKeys, (524), 137.

Krishtalka, L., \& Humphrey, P. S. (2000). Can natural history museums capture the future? BioScience, 50(7), 611-617.

Longhurst, A. R. (1998). Ecological geography of the sea. Academic Press, Orlando, Florida.

Mikkelsen, P. M., \& Cracraft, J. (2001). Marine biodiversity and the need for systematic inventories. Bulletin Marine Science, 69(2), 525-534.

Moyano, H. I., \& Larraín, A. (1976). Doraster qawashqari sp. nov. nuevo asteroideo de Chile austral (Echinodermata, Zorocallida, Zoroasteriidae). Boletín Sociedad Biología, Concepción, 50, 103-111.

Mutschke, E., \& C. Ríos. (2006). Distribución espacial y abundancia relativa de equinodermos en el estrecho de Magallanes, Chile. Ciencia y Tecnología del Mar, 29(1), 91-102.

Mutschke, E., \& Mah, C. (2009). AsteroideaStarfish. Marine Benthic Fauna of Chilean Patagonia. Nature in Focus, Santiago, Chile. Pawson, D. L., Pawson, D. J., \& King, R. A. (2010). A taxonomic guide to the Echinodermata of the South Atlantic Bight, USA: 1. Sea cucumbers (Echinodermata: Holothuroidea). Zootaxa, 2449, 1-48.

Pérez-Ruzafa, A., Alvarado, J. J., Solís-Marín, F. A., Hernández, J. C., Morata, A., Marcos, C., Barraza, E.,...Williams, S. M. (2013). Latin America echinoderm biodiversity and biogeography: Patterns and affinities. En Echinoderm research and diversity in Latin America (pp. 511-542). Springer Berlin Heidelberg.

Ríos, C., Mutschke, E., Montiel, A., Gerdes, D., \& Arntz, W. E. (2005). Soft-bottom macrobenthic faunal associations in the southern Chilean glacial fjord complex. Scientia Marina, 69(S2), 225-236.

Ríos, C., Arntz, W. E., Gerdes, D., Mutschke, E., \& Montiel, A. (2007). Spatial and temporal variability of the benthic assemblages associated to the holdfasts of the kelp Macrocystis pyrifera in the Straits of Magellan, Chile. Polar Biology, 31(1), 89-100.

Silva, N., \& Palma, S. (2008). Progress in the oceanographic knowledge of Chilean interior waters, from Puerto Montt to Cape Horn. Comité Oceanográfico Nacional.

Tommasi, L. R. (1999). Echinodermata recentes e fósseis do Brasil. Invertebrados Marinhos Registrados no Litoral Brasileiro.

Wilcox, B. A., \& Murphy, D. D. (1985). Conservation strategy: the effects of fragmentation on extinction. The American Naturalist, 125(6), 879-887.

WoRMS (2016). Echinodermata. Accessed through: World Register of Marine Species at http://www.marinespecies.org/aphia. php? $p=$ taxdetails\&id $=1806$ on 2016-0530. 
